\title{
ZEÓLITA NaA SINTETIZADA SOBRE FIBRA DE VIDRO COMO ESTRATÉGIA PARA OTIMIZAÇÃO DO ABRANDAMENTO DE ÁGUAS DURAS
}

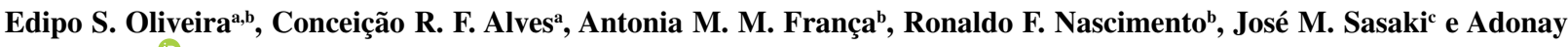 \\ R. Loiola ${ }^{\mathrm{a}, *,(1)}$ \\ aDepartamento de Química Orgânica e Inorgânica, Universidade Federal do Ceará, 60440-900 Fortaleza - CE, Brasil

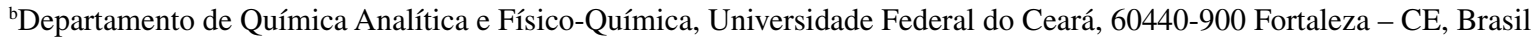 \\ 'Departamento de Física, Universidade Federal do Ceará, 60440-900 Fortaleza - CE, Brasil
}

Recebido em 07/04/2021; aceito em 06/07/2021; publicado na web em 28/07/2021

\begin{abstract}
NaA ZEOLITE SYNTHESIZED OVER FIBERGLASS AS A STRATEGY FOR OPTIMIZING HARD WATER SOFTENING. Zeolite $\mathrm{NaA}$ has been successfully synthesized over glass fiber surface previously activated by alkaline treatment. By varying the time of the treatment and the amount of silica and alumina precursors used in the reaction mixture, a sample with higher zeolite concentration could be obtained. This material was evaluated for water softening process as an alternative substitute for the use of the zeolite in its powder form, in which several drawbacks related to recovery and reuse limit its application. Water softening experiments, carried out in a continuous flow system, with a column containing the glass fiber-zeolite NaA, showed high performance in decreasing the $\mathrm{Ca}^{2+}$ ions, with complete removal achieved for $20 \mathrm{~mL}$ of simulated hard water $\left(\mathrm{Ca}^{2+} 100.0 \mathrm{mg} \mathrm{\textrm {L } ^ { - 1 }}\right)$ by using $200 \mathrm{mg}$ of the sample in form of column (10 mm diameter $\times 300 \mathrm{~mm}$ high). The sample maintained this performance in a wide range of $\mathrm{pH}(3.0-9.0)$, and also presented a feasible regenerability, with no decrease in its performance during the first four cycles of use, reaching $87.3 \%$ of efficiency over the tenth cycle.
\end{abstract}

Keywords: Zeolite A; fiber glass; hierarchical material; water softening.

\section{INTRODUÇÃO}

Embora mais de dois terços da superfície terrestre sejam recobertos por água, pouco mais de $2,5 \%$ dessa é água doce, sendo que $70 \%$ encontra-se em geleiras, $29 \%$ em reservas subterrâneas, e somente $1 \%$ em rios, lagos e zonas pantanosas. ${ }^{1}$ Apesar de ser um recurso natural renovável, o seu fornecimento abrange complexos sistemas de captura, tratamento e distribuição. Fatores como crescimento populacional, elevados índices de poluição, devastação de florestas e períodos prolongados de estiagem em diversas regiões do planeta, têm impactado fortemente a disponibilidade de água potável. ${ }^{2-4}$ Além disso, os elevados custos dos métodos convencionais de desinfecção, descontaminação e dessalinização, fazem com que cada vez mais esforços sejam empreendidos em busca do desenvolvimento de tecnologias mais eficientes e acessíveis. ${ }^{5}$

A dureza da água, propriedade relacionada à presença de altas

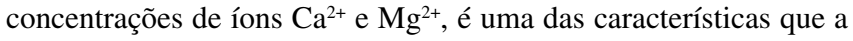
tornam imprópria para consumo, tanto doméstico como industrial. Águas subterrâneas oriundas de regiões onde há prevalência de rochas calcárias e dolomíticas, rochas sedimentares ou minerais ricos em cálcio e magnésio tendem a apresentar elevados teores desses cátions divalentes. ${ }^{6} \mathrm{O}$ tratamento da água dura, denominado de abrandamento, consiste na remoção ou substituição desses íons. Métodos de abrandamento incluem a precipitação, métodos termoquímicos, osmose inversa e processos de troca iônica. ${ }^{7}$ A troca iônica se mostra um método especialmente atrativo e oferece muitas vantagens tais como baixo custo, elevada seletividade, alta eficiência, baixos teores de resíduos gerados e, principalmente, a possibilidade de regeneração do agente ativo. ${ }^{8} \mathrm{~A}$ escolha do material a ser utilizado no processo de abrandamento via troca iônica aparece como elemento central. Nesse sentido, diversos materiais vêm sendo estudados, dentre os quais destacam-se materiais poliméricos, argilominerais e zeólitas. ${ }^{9,10}$

*e-mail: adonay@ufc.br
Zeólitas são aluminossilicatos cristalinos hidratados de estrutura aberta, constituídos por tetraedros de $\left[\mathrm{SiO}_{4}\right]^{4-}$ e $\left[\mathrm{AlO}_{4}\right]^{5-}$, ligados entre si por átomos de oxigênio e que apresentam um intricado sistema de canais e cavidades na forma de microporos (abertura $<2 \mathrm{~nm}$ ). ${ }^{11}$ Aliado a esses sistemas de poros, uma combinação única de propriedades como altas áreas superficiais e capacidade de adsorção, acidez com intensidade controlável, elevada capacidade de troca iônica e alta estabilidade térmica ${ }^{12}$ faz com que as zeólitas, particularmente as zeólitas sintéticas, encontrem emprego em inúmeras aplicações industriais importantes, compondo um mercado que atualmente gira em torno de US\$ 5,2 bilhões anuais, e que se encontra em plena expansão. ${ }^{13}$

O desempenho em aplicações como abrandamento depende fundamentalmente da capacidade de troca catiônica que, por sua vez, é maior para zeólitas com uma razão Si/Al baixa. ${ }^{14}$ A zeólita $\mathrm{NaA}$ (tipo LTA) apresenta uma razão Si/Al próxima a 1 e, portanto, se mostra como uma opção atrativa. Essa zeólita é, por sinal, a mais amplamente utilizada, em volume, e é empregada principalmente

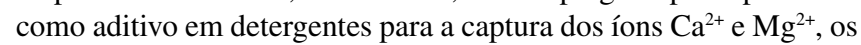
quais afetam fortemente a propriedade de detergência. ${ }^{13,15}$

Apesar das zeólitas apresentarem estruturas cristalinas com sistemas de poros bem definidos, a inexistência de poros maiores impõe limitações difusionais importantes, principalmente se levarmos em consideração que as zeólitas são materiais policristalinos, isso é, apresentam-se na forma de pó. ${ }^{16}$ Para algumas aplicações práticas, portanto, uma compactação das zeólitas, geralmente na forma de pellets, se faz necessária. Porém, isso acaba restringindo o acesso das espécies de interesse aos sítios ativos dos cristais zeolíticos presentes na superfície dos pellets. Uma estratégia que vem ganhando destaque para superar tais limitações envolve o conceito de hierarquização de estruturas zeolíticas. ${ }^{17}$ Ela combina os benefícios da baixa aglomeração de partículas, promovendo otimização da difusão de adsorbatos, com a redução de perdas observadas nas etapas de recuperação (filtração, centrifugação etc.) $)^{18,19}$ 
A hierarquização é uma propriedade recorrente na natureza e são muitos os exemplos de organismos e estruturas que apresentam redes de poros interconectados para um transporte de massa eficiente em termos energéticos. ${ }^{20}$ São conhecidas diversas metodologias para o preparo de zeólitas hierárquicas, podendo-se destacar rotas hidrotérmicas que se valem da cristalização de finas camadas de zeólita em suportes inertes ou reativos como alumina, aço, carbono, sílica, vidro, polímeros e diatomita. ${ }^{20,21}$ Nesse sentido, uma alternativa promissora e de baixo custo envolve o uso de fibras cerâmicas que além de atuarem como suporte para a cristalização da zeólita, possam também servir como fonte de $\mathrm{Si}$ e $\mathrm{Al}^{22,23}$

No presente trabalho, apresentamos o desenvolvimento de um material poroso hierarquicamente estruturado, preparado a partir do crescimento de cristais da zeólita $\mathrm{NaA}$ sobre a superfície de fibras de vidro, e o estudo da avaliação do seu potencial no processo de abrandamento de água. Levando-se em conta que a preparação de zeólitas hierárquicas é um processo oneroso que, em geral, demanda diversas etapas e envolve o uso de reagentes químicos nocivos, a metodologia de síntese proposta neste trabalho se destaca por aliar o uso de matéria prima de baixo custo com procedimentos experimentais simples, dando origem a um material zeolítico com estrutura hierarquizada adequado para o emprego no abrandamento de água dura em sistemas de fluxo.

\section{PROCEDIMENTOS EXPERIMENTAIS}

\section{Síntese da zeólita $\mathrm{NaA}$ suportanda em fibra de vidro}

Fibra de vidro (FV) (Synth, 99,5\% de $\mathrm{SiO}_{2}$ ) foi utilizada como suporte para síntese da zeólita NaA. A fibra foi incialmente submetida a tratamento alcalino objetivando a geração de rugosidade para, assim, direcionar a nucleação de zeólita e seu subsequente crescimento sobre a superfície da primeira. $\mathrm{O}$ procedimento consistiu na imersão de $10,0 \mathrm{~g}$ da amostra FV em 100,0 mL de solução de $\mathrm{NaOH} \mathrm{4,0} \mathrm{mol} \mathrm{L}{ }^{-1}$, utilizando frasco de polipropileno, e agitação orbital moderada (200 rpm) durante períodos de $24,0 \mathrm{~h}$ ou $72,0 \mathrm{~h}$. Após o tratamento alcalino, os materiais ativados foram lavados primeiro com solução de $\mathrm{HCl}$ 1,0 mol L ${ }^{-1}(2 \times)$, depois com água destilada (2×) e, em seguida, seco em estufa a $80,0^{\circ} \mathrm{C}$ por $24 \mathrm{~h}$. Os materiais obtidos, isso é, as fibras de vidro ativadas, foram identificados como FVA-24 e FVA-72, de acordo com os tempos utilizadas no tratamento de 24,0 h e 72,0 h, respectivamente.

As amostras de fibras ativadas foram submetidas a tratamento hidrotérmico na presença de diferentes quantidades de uma solução precursora. Para a preparação da solução precursora, 7,16 g de $\mathrm{Na}_{2} \mathrm{SiO}_{3}$ (Sigma-Aldrich, 50-53\% de $\mathrm{SiO}_{2}$ ) foram dissolvidos em 35,0 mL de $\mathrm{NaOH} 0,21 \mathrm{~mol} \mathrm{~L}^{-1}$, usando um béquer de polipropileno, e em outro béquer 5,00 g de $\mathrm{NaAlO}_{2}$ (Sigma-Aldrich, 50-56\% de $\mathrm{Al}_{2} \mathrm{O}_{3}$ ) foram dissolvidos em 35,0 $\mathrm{mL}$ de $\mathrm{NaOH} 0,21 \mathrm{~mol} \mathrm{~L}^{-1}$. A mistura dessas duas soluções resultou na solução precursora. Em seguida, 8,0 g das amostras das fibras ativadas (FVA-24 e FVA-72) foram acondicionadas em um reator de Teflon, com capacidade de $72,0 \mathrm{~mL}$, juntamente com a solução precursora com o volume do reator completo (sistema A) ou pela metade (sistema B). Em ambos os casos, as fibras de vidro ficaram totalmente submersas na solução. $\mathrm{O}$ reator foi confinado em autoclave de aço inox, mantido em repouso a $25,0^{\circ} \mathrm{C}$ por $18,0 \mathrm{~h}$ e, em seguida, aquecido a $100{ }^{\circ} \mathrm{C}$ por $4 \mathrm{~h}$, em estufa. Após resfriamento, o material obtido foi removido do reator, lavado com água destilada e centrifugado a $4000 \mathrm{rpm}$ (processo repetido 7 vezes, com intuito de reduzir a alcalinidade do meio). Cristais de zeólita que foram gerados sem estarem incorporados às fibras foram removidos usando-se jatos de água sobre as amostras, dispostas em uma peneira que foi usada com anteparo. As amostras obtidas foram denominadas de ZH-24-A,
ZH-24-B, ZH-72-A e ZH-72-B e uma representação esquemática do processo de síntese é apresentado na Figura 1.

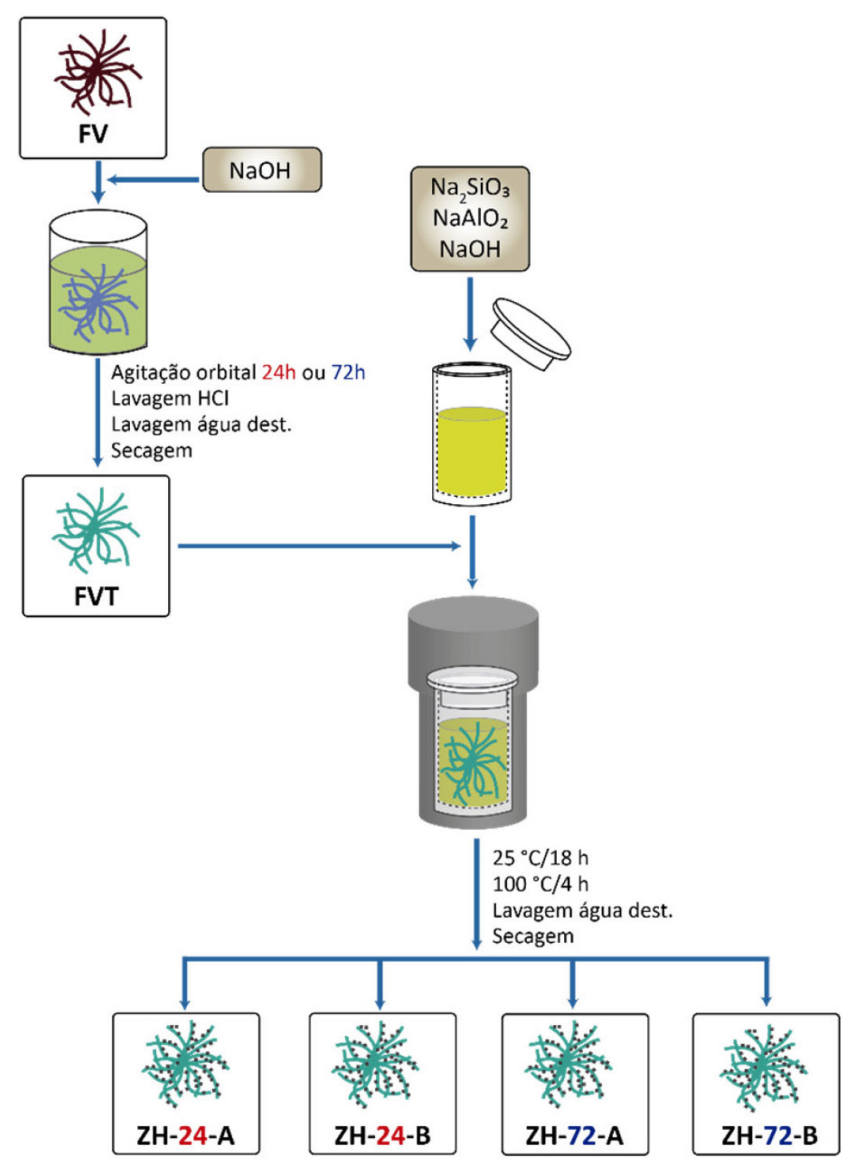

Figura 1. Representação simplificada dos procedimentos para a preparação das zeólitas hierárquicas

\section{Caracterização dos materiais}

Difração de raios $X$

As medidas de difração de raios $X$ foram conduzidas em difratômetro para amostras policristalinas modelo X-Pert PRO MPDPanalytical, com radiação de $\mathrm{Co}-\mathrm{K} \alpha$ proveniente de tubo de cobalto operado com foco linear a $40,0 \mathrm{kV}$ e $40,0 \mathrm{~mA}$. Os difratogramas foram obtidos no intervalo de $2 \theta=5,0-50,0^{\circ}$ e tempo de contagem de $67,0 \mathrm{~s}$. A identificação de fases cristalinas foi realizada por meio do software X-Pert HighScore Plus (Panalytical).

\section{Microscopia eletrônica de varredura}

Análises de microscopia eletrônica de varredura foram realizadas em microscópio Quanta FEG, modelo 450-FEI, utilizando feixe de elétrons com aceleração de $20,0 \mathrm{kV}$ e detectores de elétrons secundários e de energia dispersiva de raios $\mathrm{X}$. As amostras foram preparadas em suportes de alumínio utilizando fita de carbono duplaface e metalizadas com uma fina camada de ouro (aproximadamente $20 \mathrm{~nm}$ ) em equipamento Quorum Q150T ES.

\section{Espectroscopia na região do infravermelho}

Análises de espectroscopia vibracional na região do infravermelho foram realizadas com todas as amostras sintetizadas, bem como com os suportes de vidro sem tratamento alcalino ou zeólita incorporada. As análises foram realizadas com as amostras dispersas em $\mathrm{KBr}$, na forma de pastilhas, em equipamento Shimadzu, modelo IRTracer-100, na faixa de 400 a $4000 \mathrm{~cm}^{-1}$. 


\section{Fluorescência de raios $X$}

Para a determinação da composição química das amostras deste estudo, foram realizadas medidas de fluorescência de raios X, utilizando espectrômetro de raios $\mathrm{X}$ de dispersão de comprimento de onda (WDX) sequencial da Rigaku, modelo ZSX mini II, operando a $40 \mathrm{kV}$ e $1,2 \mathrm{~mA}$, com tubo de $\mathrm{Pd}$, capaz de produzir análises semiquantitativas dos elementos de número atômico maior ou igual ao do elemento flúor, até o elemento urânio.

\section{Ensaios de abrandamento}

Um sistema em coluna, utilizando a amostra contendo zeólita cuja síntese se mostrou mais satisfatória, foi montado para a realização dos ensaios de abrandamento. As amostras de fibra recobertas com a zeólita $\mathrm{NaA}$ foram acondicionadas em coluna de vidro de borossilicato através da qual se passou solução de $\mathrm{Ca}^{2+} 100,0 \mathrm{mg} \mathrm{L}^{-1}$.

Para o estudo da influência da massa de zeólita hierárquica, foram utilizadas colunas ( $10 \mathrm{~mm}$ de diâmetro $\times 300 \mathrm{~mm}$ de altura) contendo 25,$0 ; 50,0 ; 75,0 ; 100,0 ; 200,0 ; 300,0 ; 400,0$ e 500,0 mg do material. A cada coluna passou-se alíquotas de $20,0 \mathrm{~mL}$ da solução de $\mathrm{Ca}^{2+}$

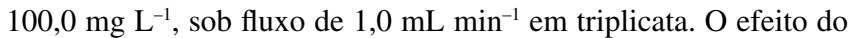
$\mathrm{pH}$ na adsorção de íons cálcio foi avaliado ajustando-se o $\mathrm{pH}$ para 3,$0 ; 5,0 ; 7,0$ ou 9,0. Nesses experimentos, foram eluídas alíquotas de $100 \mathrm{~mL}$ da solução de $\mathrm{Ca}^{2+} 100 \mathrm{mg} \mathrm{L}^{-1}$, gota a gota, fazendo-se coletas de 5,0 mL após a eluição.

Para o estudo da capacidade de regeneração do suporte, foi feita a eluição de alíquotas de $20,0 \mathrm{~mL}$ da solução de $\mathrm{Ca}^{2+} 100,0 \mathrm{mg} \mathrm{L}^{-1}$ em 500,0 mg do material zeolítico. Ao término de cada eluição, foi realizada regeneração por meio da passagem de $100,0 \mathrm{~mL}$ de uma solução de $\mathrm{NaCl} 1000,0 \mathrm{mg} \mathrm{L}^{-1}$ e de e $50,0 \mathrm{~mL}$ de água bidestilada. Ao todo, foram feitas 9 regenerações, totalizando 10 ciclos de abrandamento.

Embora o $\mathrm{Ca}^{2+}$ seja o íon mais representativo quando se trata de dureza de água, outros íons como, por exemplo, $\mathrm{Mg}^{2+} \mathrm{e} \mathrm{Fe}^{2+}$ também podem estar presentes. Com o intuito de avaliar o desempenho dos materiais sintetizados em um cenário mais próximo ao de amostras reais, foram preparadas três soluções de $\mathrm{Ca}^{2+}$ e de $\mathrm{Mg}^{2+}$ nas concentrações de $\mathrm{Ca}^{2+} 103,8 \mathrm{mg} \mathrm{L}^{-1}, \mathrm{Mg}^{2+} 37,2 \mathrm{mg} \mathrm{L}^{-1}$ (3:1); $\mathrm{Ca}^{2+} 32,5 \mathrm{mg} \mathrm{L}^{-1}, \mathrm{Mg}^{2+} 105,8 \mathrm{mg} \mathrm{L}^{-1}$ (1:3) e Ca ${ }^{2+} 104,5 \mathrm{mg} \mathrm{L}^{-1}$, $\mathrm{Mg}^{2+} 103,6 \mathrm{mg} \mathrm{L}^{-1}$ (1:1). Alíquotas de 20,0 $\mathrm{mL}$ dessas soluções foram eluídas em colunas contendo $500 \mathrm{mg}$ de amostras de fibra recobertas com a zeólita NaA. Além disso, foi também realizado o tratamento com amostras de água de dois poços artesianos, localizados nas cidades de Tauá (Sítio Bomtempo, 554'16.3”'S 40²6’16.5”W, com $164 \mathrm{~m}$ de profundidade, sendo $130 \mathrm{~m}$ no aluvião e $34 \mathrm{~m}$ no cristalino) e Crateús (Sítio Calcanha, 5²6'06.0”S 4054'06.3”W, com 80 m de profundidade, sendo $4 \mathrm{~m}$ no aluvião e $76 \mathrm{~m}$ no cristalino), ambas no estado do Ceará.

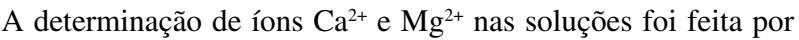
meio de titulação volumétrica de complexação. As soluções foram alcalinizadas $(\mathrm{pH} 10,0)$ e tituladas com solução padrão de ácido etilenodiaminatetracético (EDTA). O ponto de equivalência foi determinado com o auxílio dos indicadores complexantes Eriocromo $\mathrm{T}$, para determinação de dureza total $\left(\mathrm{Ca}^{2+} \mathrm{e} \mathrm{Mg}^{2+}\right)$, e Murexida para determinação de íons $\mathrm{Ca}^{2+}$.

\section{RESULTADOS E DISCUSSÃO}

\section{Caracterização}

A seguir são apresentados e discutidos os resultados de caracterização dos materiais zeolíticos juntamente com a avaliação da aplicabilidade desses materiais no abrandamento de água por meio de processo em fluxo.

As composições das amostras objeto desse estudo são mostradas na Tabela 1. Os resultados são apresentados na forma equivalente de óxidos.

A fibra de vidro (FV) apresenta como componente majoritário o óxido de silício. Convém ressaltar que a conversão para a forma de óxido é uma prática comum em medidas de fluorescência de raios $\mathrm{X}$, não significando que seja essa a forma presente na amostra. Contudo, este formato permite avaliar mudanças composicionais relacionadas à formação (ou não) das fases de interesse. As amostras de materiais zeolíticos apresentam teores elevados de óxido de alumínio na sua composição, o que é um forte indicativo da formação de zeólita. Quando se compara a quantidade de alumínio e silício entre a amostra de zeólita pura e a amostra ZH-72-A, por exemplo, e admitindo-se que quase a totalidade do alumínio faz parte da estrutura da zeólita, é possível estimar que a quantidade de zeólita na amostra final, portanto, incorporada à superfície da fibra de vidro, aproxima-se de $50 \%$, em massa. Além disso, observa-se a inexistência de compostos que possam estar associados à formação de impurezas, exceto alguns elementos já presentes na fibra de vidro.

\section{Fibra de vidro}

O tratamento alcalino da fibra de vidro teve por objetivo modificar sua superfície gerando rugosidade que pudesse favorecer a nucleação e subsequente crescimento da zeólita $\mathrm{NaA}$, incorporando-a na superfície do suporte, composto essencialmente por sílica.

Os difratogramas de raios $\mathrm{X}$ dessas amostras (Figura 2a) demonstram que o tratamento alcalino não promoveu alterações estruturais nas características já amorfas dos materiais, não havendo, portanto, nesse processo a formação de fases cristalinas. As micrografias da fibra de vidro são mostradas na Figura $2 b$. Por meio dessas imagens é possível verificar que a fibra de vidro sem tratamento (amostra FV) apresenta uma superfície predominantemente lisa e com textura uniforme, enquanto a exposição ao meio básico dá origem a deformações superficiais, isto é, uma rugosidade discreta que é percebida na forma de pequenas crateras na amostra FVA-24 e que se torna mais evidente na amostra FVA-72, cujo tempo de contato com a solução de $\mathrm{NaOH}$ foi maior. Nas análises de espectroscopia de absorção na região do infravermelho das amostras FVA-24 e

Tabela 1. Composição química das amostras, em porcentagem em massa, determinada por fluorescência de raios $X$

\begin{tabular}{|c|c|c|c|c|c|c|}
\hline Óxido/Amostra & FV & ZH-24-A & ZH-24-B & ZH-72-A & ZH-72-B & Zeólita A \\
\hline $\mathrm{Al}_{2} \mathrm{O}_{3}$ & 1,63 & 18,35 & 19,05 & 22,29 & 17,61 & 30,22 \\
\hline $\mathrm{SiO}_{2}$ & 82,26 & 78,74 & 78,30 & 75,27 & 78,23 & 55,25 \\
\hline $\mathrm{Na}_{2} \mathrm{O}$ & 6,97 & - & - & - & - & 14,53 \\
\hline $\mathrm{MgO}$ & 4,44 & - & - & - & - & - \\
\hline $\mathrm{Cl}$ & 0,16 & - & 0,14 & - & 0,75 & - \\
\hline $\mathrm{K}_{2} \mathrm{O}$ & 2,63 & 1,65 & 1,83 & 1,82 & 2,07 & - \\
\hline $\mathrm{Fe}_{2} \mathrm{O}_{3}$ & 1,50 & 1,20 & 0,68 & 0,56 & 1,33 & - \\
\hline
\end{tabular}



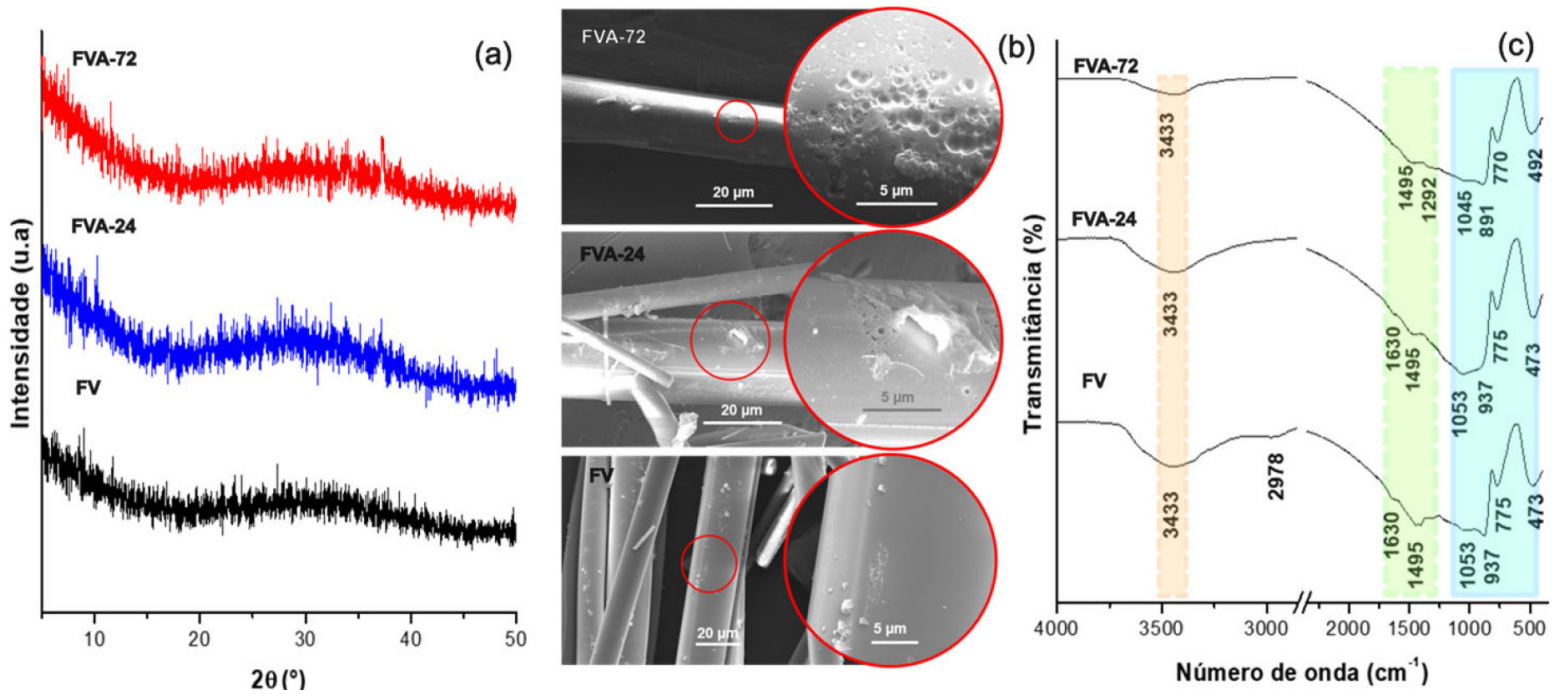

Figura 2. Caracterização dos materiais hierárquicos contendo zeólita A, sem tratamento alcalino prévio da fibra de vidro: (a) DRX, (b) MEV e (c) IV

FVA-72, cujos espectros são mostrados na Figura 2c, destacam-se as bandas centradas em 1053, 775 e $473 \mathrm{~cm}^{-1}$, para a amostra FVA-24, e as bandas 1045, 770 e $492 \mathrm{~cm}^{-1}$, para a amostra FVA-72, as quais correspondem à ligação $\mathrm{Si}-\mathrm{O}-\mathrm{Si}$ dos silicatos que compõem a estrutura das fibras de vidro.

\section{Zeólita hierárquicas}

Os difratogramas de raios $\mathrm{X}$ das amostras $\mathrm{ZH}-24 \mathrm{~A}$ e $\mathrm{ZH}-24 \mathrm{~B}$, obtidas a partir das fibras submetidas a tratamento alcalino de $24 \mathrm{~h}$, são apresentados na Figura 3a. Em ambas as amostras são observados picos condizentes com a zeólita $\mathrm{NaA}$, identificadas pela microficha do banco de dados ICSD 24901, porém, a amostra preparada com menor quantidade de reação precursora (ZH-24B) apresenta picos menos intensos que o observado para a amostra sintetizada com maior volume da solução precursora (ZH-24A), o que sugere menor quantidade de cristais de zeólita sobre a superfície da sílica amorfa (fibra de vidro). As micrografias para essas duas amostras são mostradas na Figura 3b, na qual é possível observar facilmente a formação de cristais com hábito cúbico sobre a superfície da fibra de vidro, com prevalência em regiões com rugosidade ou com falhas aparentes. Para essas amostras, não é possível identificar diferença apreciável quanto a influência do meio reacional (A ou B) em relação a eficiência da síntese dos cristais zeolíticos uma vez que a formação desses se mostra muito semelhante nas duas amostras.
As análises de espectroscopia de absorção na região do infravermelho fornecem informações que auxiliam na avaliação estrutural dos materiais obtidos, servindo como uma importante técnica complementar à difração de raios $\mathrm{X}$. O espectro de infravermelho da amostra ZH-24-A (Figura 3c) apresenta um conjunto de sinais intensos característicos da zeólita NaA. As bandas em 3431 e $1655 \mathrm{~cm}^{-1}$ referem-se ao estiramento simétrico da ligação O-H de moléculas de água ou de grupos silanois. ${ }^{24} \mathrm{~A}$ banda intensa em $1005 \mathrm{~cm}^{-1}$ é atribuída ao estiramento assimétrico da ligação ( $\left.\mathrm{Si}, \mathrm{Al}\right)-\mathrm{O}$, enquanto a banda discreta em $667 \mathrm{~cm}^{-1}$ diz respeito ao estiramento simétrico da ligação ( $\mathrm{Si}, \mathrm{Al})-\mathrm{O} \cdot{ }^{25-27} \mathrm{~A}$ banda em $555 \mathrm{~cm}^{-1}$ é relacionada ao anel duplo de quatro membros (4DR) que conecta as unidades sodalita, e a banda em $465 \mathrm{~cm}^{-1}$ refere-se às ligações $\mathrm{Si}-\mathrm{O}-\mathrm{Al} .{ }^{28}$ Estes sinais mostram-se com uma forte evidência da formação da zeólita A. Embora algumas dessas bandas sejam visíveis no espectro da amostra ZH-24-B, elas apresentam intensidades consideravelmente menores e, além disso, outras bandas estão presentes, apontando uma formação limitada da zeólita NaA nesta amostra.

Com o intuito de obter uma maior quantidade de zeólita, foi feita a síntese de mais material agora usando fibra de vidro tratada em meio alcalino por um período maior, de $72 \mathrm{~h}$. Os resultados de caracterização para as amostras obtidas são apresentados na Figura 4. Os difratogramas de raios $\mathrm{X}$ das amostras ZH-72-A e ZH-72-B (Figura 4a) apresentam picos característicos da zeólita $\mathrm{NaA}$ e mais
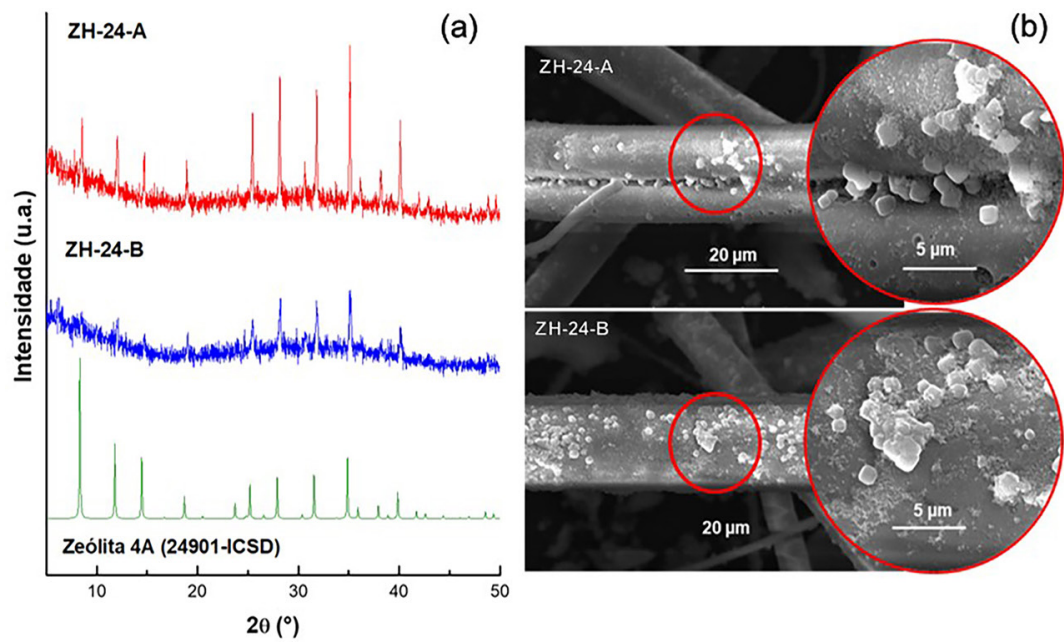

(b)

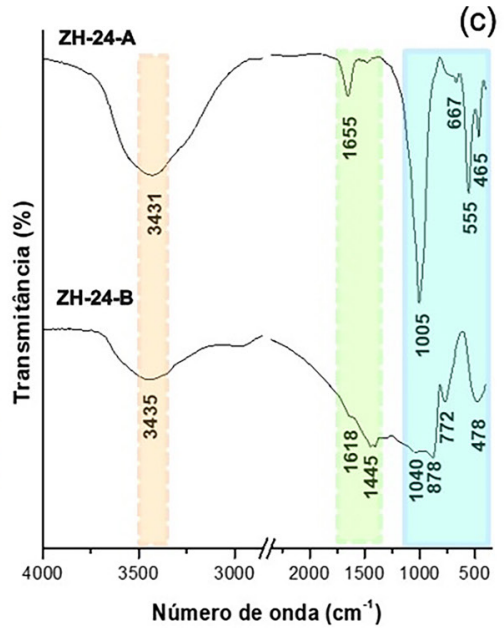

Figura 3. Caracterização dos materiais contendo a zeólita A, após 24 de tratamento alcalino da fibra de vidro: (a) DRX, (b) MEV e (c) IV 

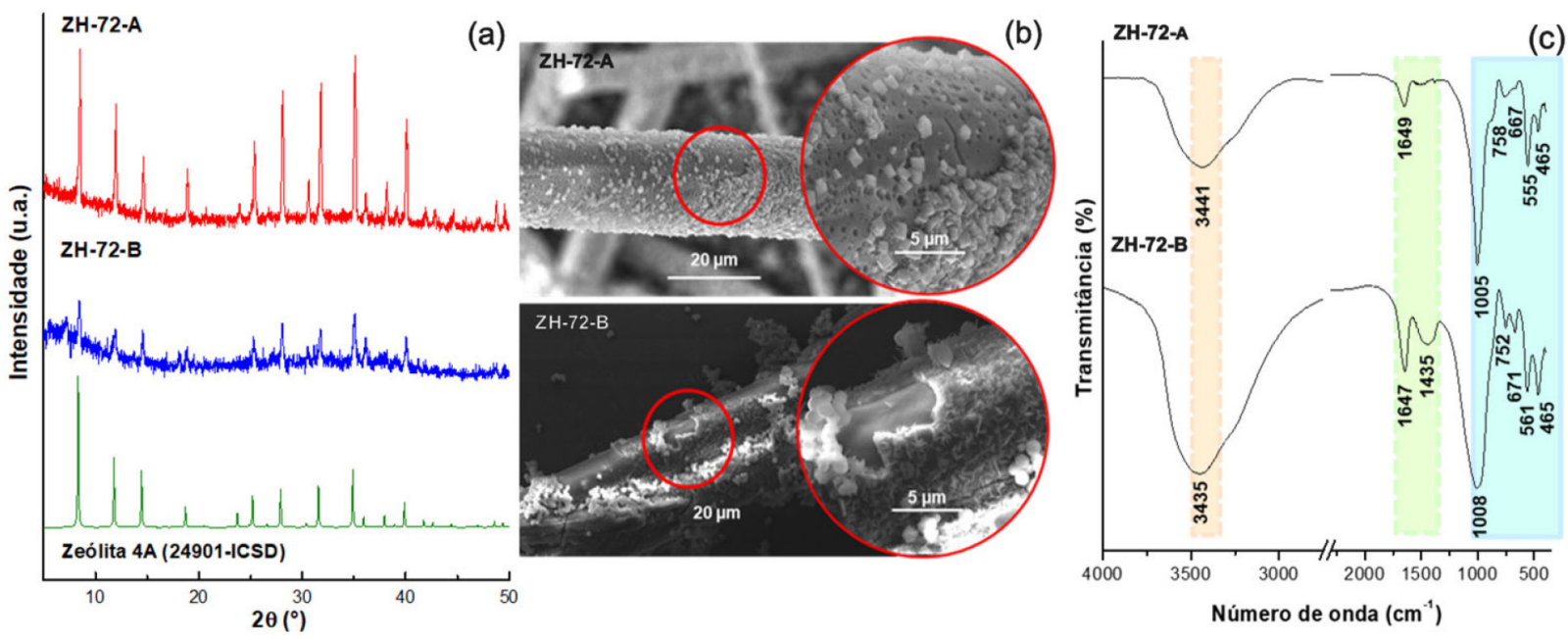

Figura 4. Caracterização dos materiais contendo zeólita A, após $72 h$ de tratamento alcalino da fibra de vidro: (a) DRX, (b) MEV e (c) IV

intensos que os observados para as amostras preparadas a partir das fibras submetidos ao tratamento alcalino de $24 \mathrm{~h}$. Além disso, é importante mencionar que não foram observados picos de difração relacionadas a outras fases cristalinas, um forte indicativo de eficiência das sínteses.

As micrografias dessas amostras (Figura 4b) permitem observar os cristais cúbicos da zeólita $\mathrm{NaA}$, como apontado nos resultados de DRX, formando uma camada relativamente coesa sobre a superfície da fibra de vidro. Uma propriedade importante observada, e que é desejável, diz respeito ao favorecimento da cristalização da zeólita nas rugosidades da fibra de vidro, fazendo com que os cristais zeolíticos se mantenham ligados ao suporte e, ao mesmo tempo, não se organizem de forma aglomerada. Essas características são especialmente relevantes para o processo de abrandamento da água, pois maximiza o contato entre os íons $\mathrm{Ca}^{2+}$ presentes na água dura a ser tratada e os cristais da zeólita. Os espectros do IV, em particular da amostra ZH-72-A, mostram sinais intensos e coerentes com a zeólita NaA, como já discutido para as outras amostras.

\section{Abrandamento}

Tendo em vista os resultados observados para a amostra $\mathrm{ZH}$ 72-A, com a zeólita A apresentando cristais com tamanhos regulares e recobrindo de forma razoavelmente satisfatória o suporte de vidro, esta amostra foi escolhida para os ensaios de abrandamento. $\mathrm{Na}$ Figura 5 são apresentados os resultados dos ensaios de abrandamento em função da massa do material hierárquico. $\mathrm{O}$ material mostrou-se

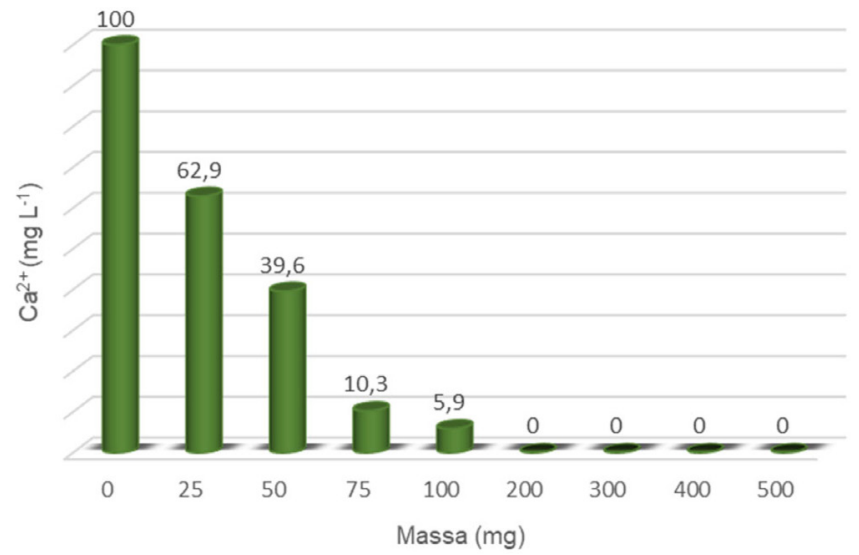

Figura 5. Efeito da variação de massa da amostra ZH-72-A nos ensaios de abrandamento eficiente no processo de abrandamento e, como esperado, apresentou aumento na eficiência de remoção de íons $\mathrm{Ca}^{2+}$ com o aumento da massa de material zeolítico utilizado, com abrandamento completo sendo atingido a partir de $200,0 \mathrm{mg}$.

Considerando-se os resultados apresentados na Figura 5, a capacidade de remoção para íons $\mathrm{Ca}^{2+}$ é estimada em $10 \mathrm{mg}$ por $\mathrm{g}$ de material zeolítico. Como aproximadamente metade da massa do material zeolítico é de fato a zeólita $\mathrm{A}$, conforme previsto nas análises de fluorescência de raios $\mathrm{X}$, a capacidade de remoção para íons $\mathrm{Ca}^{2+}$ se aproxima de $20 \mathrm{mg}$ por g de zeólita. Esse valor é um pouco abaixo dos valores observados em outros trabalhos que fizeram uso da zeólita A na forma de pó, ${ }^{27-30}$ o que de certo modo é esperado já que os cristais zeolíticos dos materiais aqui apresentados se encontram integrados à superfície da fibra de vidro e portanto têm parte da sua superfície inacessível. Contudo, a praticidade advinda da possibilidade de se usar um sistema em fluxo pode compensar essa diferença. Outra propriedade importante para um agente de abrandamento é sua capacidade de regeneração e reuso. Nesse sentido, foram realizados ensaios de regeneração percolando-se uma solução $\mathrm{Na}^{+}\left(1000,0 \mathrm{mg} \mathrm{L}^{-1}\right)$ através da coluna contendo o material zeolítico. É sabido que soluções com alta concentração deste íon (cerca de 10× a concentração de $\mathrm{Ca}^{2+}$ ) apresenta potencial para substituir os íons $\mathrm{Ca}^{2+}$ então retidos na estrutura da zeólita, reativando-a para o processo de abrandamento ${ }^{31}$ A Figura 6 mostra a eficiência de regeneração e reuso da coluna em alíquotas de $20,0 \mathrm{~mL}$ da solução de $\mathrm{Ca}^{2+} 100,0 \mathrm{mg} \mathrm{L}^{-1}$, usando 500,0 mg do material zeolítico, em sucessivos ciclos.

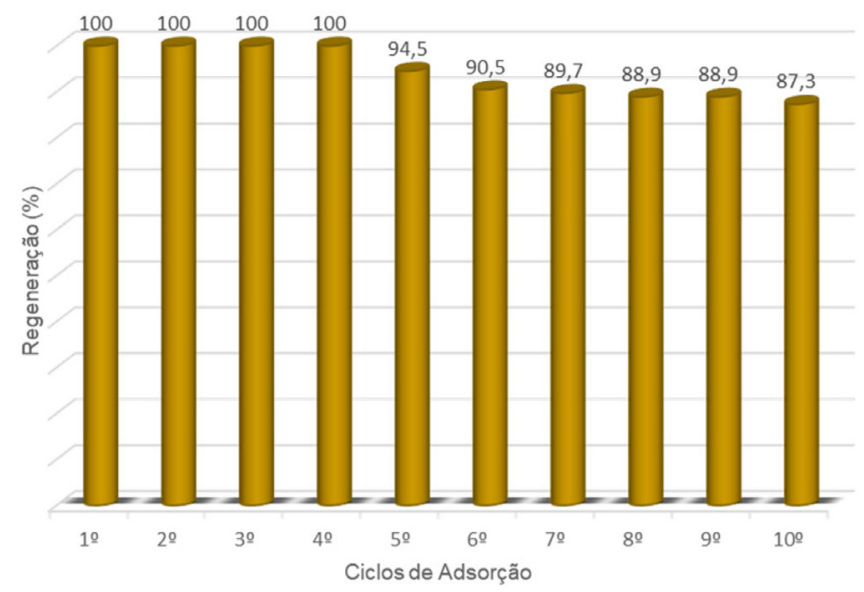

Figura 6. Desempenho da zeólita hierárquica ZH-72-A no processo de abrandamenteo em sucessivos ciclos de regeneração 
Nos primeiros quatro ciclos, os processos de regeneração levaram ao abrandamento total da água utilizada, apresentando uma ligeira queda a partir do quinto ciclo, com abrandamento de $94,5 \%$ da água. Observou-se uma queda contínua na eficiência, porém pequena, nos ciclos seguintes até o décimo ciclo, no qual a eficiência foi de $87,3 \%$. Esse decréscimo na eficiência está possivelmente associado à lixiviação de cristais de zeólita provocada pela solução em fluxo. Entretanto, tal decréscimo pode ser considerado aceitável considerando-se a quantidade ciclos avaliados.

A Figura 7 mostra os resultados da concentração remanescente de $\mathrm{Ca}^{2+}$ após tratamento, sob fluxo contínuo, na coluna contendo a amostra ZH-72-A (500,0 mg), com a coleta de alíquotas de 5,0 mL. Observa-se que conforme a solução de $\mathrm{Ca}^{2+}$ passa através da coluna, o teor de $\mathrm{Ca}^{2+}$ remanescente aumenta, indicando a saturação crescente do material zeolítico. Com a passagem do volume total de $100,0 \mathrm{~mL}$ de solução de $\mathrm{Ca}^{2+} 100 \mathrm{mg} \mathrm{L}{ }^{-1}$, verificou-se uma concentração remanescente de $46 \mathrm{mg} \mathrm{L}^{-1}$, o que corresponde, para esse volume, a uma capacidade de abrandamento de $54 \%$.

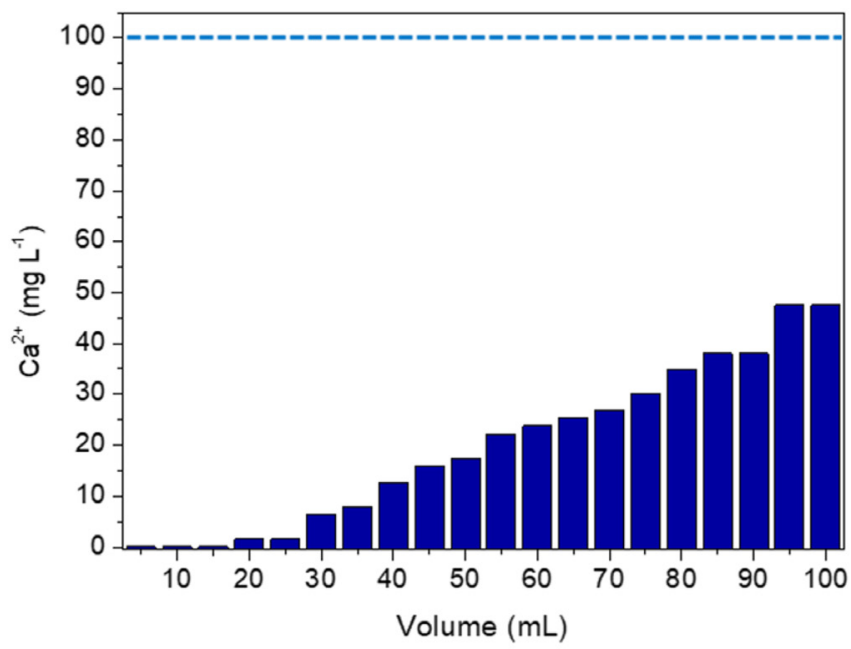

Figura 7. Concentração de $\mathrm{Ca}^{2+}$ após passagem de solução de $\mathrm{Ca}^{2+}$ 100,0 mg $L^{-1}$ por coluna de zeólita hierárquica

A avaliação do efeito do pH sobre a eficiência da amostra de zeólita hierárquica mostra-se relevante considerando as diferentes condições encontradas para amostras reais, notadamente águas de poço que se apresentam em uma faixa ampla de $\mathrm{pH} .{ }^{32} \mathrm{~A}$ influência do $\mathrm{pH}$ sobre a eficiência da amostra $\mathrm{ZH}-72-\mathrm{A}$ como abrandador foi estudada em valores de $\mathrm{pH}$ de 3,0, 5,0, 7,0 e 9,0. As concentrações remanescentes de $\mathrm{Ca}^{2+}$ após a passagem das respectivas soluções pela coluna são apresentadas na Figura 8, para cada valor de $\mathrm{pH}$.

Observa-se uma completa remoção dos íons $\mathrm{Ca}^{2+}$ para o volume de solução tratado $(20,0 \mathrm{~mL})$ considerando-se os quatro valores de pH analisados, o que implica em não haver variação na eficiência do processo de abrandamento, com elevada eficiência do material zeolítico independemente do $\mathrm{pH}$ estudado.

Os resultados de abrandamento utilizando soluções contendo $\mathrm{Ca}^{2+}$ e $\mathrm{Mg}^{2+}$, assim como das amostras de poço são apresentados na Figura 9.

Conforme pode ser observado na Figura 9a, o tratamento realizado para a solução na proporção 3:1 $\left(\mathrm{Ca}^{2+}\right.$ e $\left.\mathrm{Mg}^{2+}\right)$ fez com que as concentrações de $\mathrm{Ca}^{2+}$ e $\mathrm{Mg}^{2+}$ decrescessem de 103,75 e $37,15 \mathrm{mg} \mathrm{L}^{-1}$ para 1,58 e $41,38 \mathrm{mg} \mathrm{L}^{-1}$, indicando que o material zeolítico mostra-se mais seletivo para o íon $\mathrm{Ca}^{2+}$. O tratamento da solução com proporção invertida, isso é, 1:3, em que a concentração do íon $\mathrm{Mg}^{2+}$ é maior, deixa ainda mais claro o efeito da seletividade para o íon $\mathrm{Ca}^{2+}$, e que é reforçado no tratamento da solução $1: 1$.

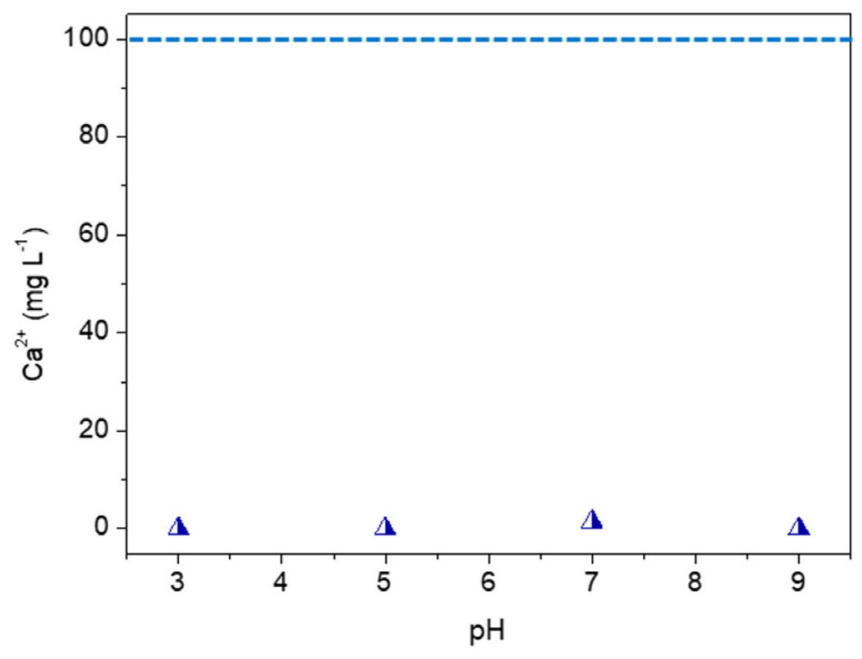

Figura 8. Efeito do pH da solução no desempenho da amostra ZH-72-A no abrandamento
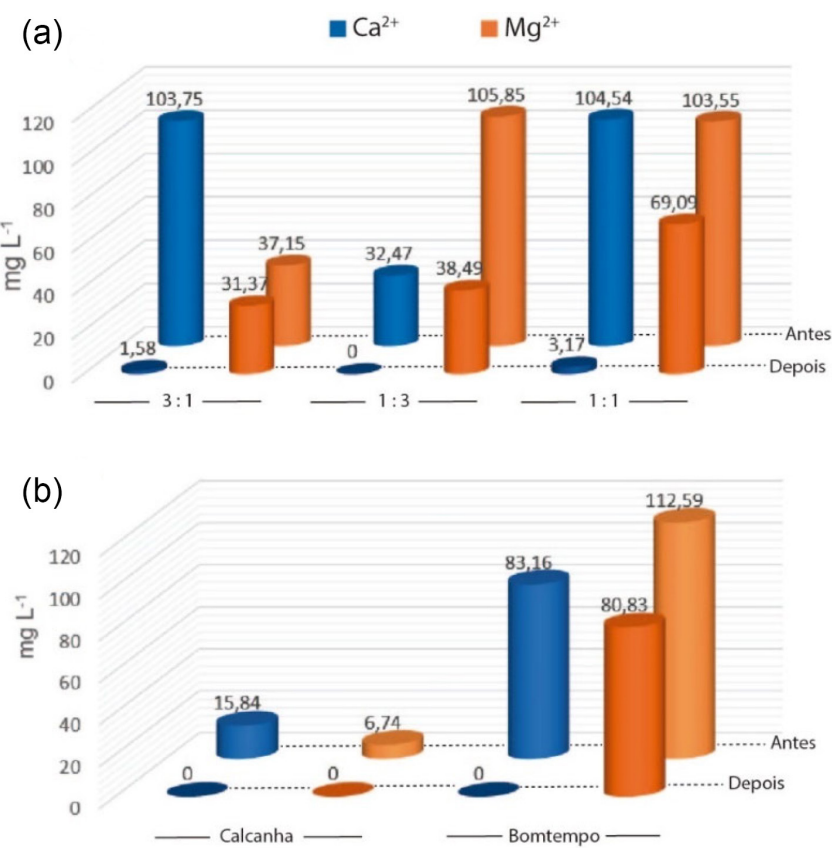

Figura 9. Concentração de $\mathrm{Ca}^{2+}$ e de $\mathrm{Mg}^{2+}$ (a) em soluções com razões 1:3, 1:1 e 1:3, e (b) em amostras de poços artesianos antes e após passagem por coluna de zeólita hierárquica

Esses resultados estão de acordo com o reportado por Ghadamnan et al. ${ }^{33}$ e se justificam pela maior afinidade entre os íons $\mathrm{Ca}^{2+}$ e a estrutura da zeólita A, cuja abertura de poros apresenta-se mais próxima ao raio iônico hidratado do $\mathrm{Ca}^{2+}(0,412 \mathrm{~nm})$ que o do $\mathrm{Mg}^{2+}(0,428 \mathrm{~nm}) \cdot{ }^{34,35}$ Ao comparar as concentrações dos íons $\mathrm{Ca}^{2+} \mathrm{e}$ $\mathrm{Mg}^{2+}$ nas duas amostras de água de poço (Figura 9b), antes e após o tratamento com o material zeolítico, foi observado que na amostra 1 (poço Calcanha), os íons $\mathrm{Ca}^{2+} \mathrm{e} \mathrm{Mg}^{2+}$ foram totalmente removidos da amostra, o que era esperado considerando-se a baixa concentração desses íons em solução $\left(15,84 \mathrm{mg} \mathrm{L}^{-1}\right.$ para $\mathrm{Ca}^{2+}$ e $6,65 \mathrm{mg} \mathrm{L}^{-1}$ para $\mathrm{Mg}^{2+}$ ). Para a amostra 2 (poço Bomtempo), com concentrações de íons $\mathrm{Ca}^{2+}$ e $\mathrm{Mg}^{2+}$ de 83,6 e 111,2 $\mathrm{mg} \mathrm{L}^{-1}$, respectivamente, os íons $\mathrm{Ca}^{2+}$ foram adsorvidos em sua totalidade e a concentração de íons $\mathrm{Mg}^{2+}$ em solução foi reduzida a $79,83 \mathrm{mg} \mathrm{L}^{-1}$, resultados que estão de acordo com ensaios realizados nas amostras preparadas em laboratório. 


\section{CONCLUSÕES}

A síntese da zeólita NaA sobre uma estrutura filamentosa de vidro de baixo custo se mostrou eficiente, com cristais zeolíticos crescidos presos à superfície do vidro. Essa característica demandou tratamento alcalino, com o tempo de $72 \mathrm{~h}$, levando a um material com rugosidade apreciável, porém, sem comprometer sua estrutura. Esse tipo de material zeolítico propiciou o uso de colunas para processo de abrandamento de água dura, realizado sob fluxo, tomando-se como referência uma solução de $\mathrm{Ca}^{2+}$ com concentração condizente com água muito dura. $\mathrm{O}$ material apresentou elevada seletividade para $\mathrm{Ca}^{2+}$ na presença de íon $\mathrm{Mg}^{2+}$, assim como se mostrou adequado para o abrandamento de amostras de água de poços artesianos, apresentado resultados promissores e com potencial de regeneração.

\section{AGRADECIMENTOS}

Os autores agradecem à Central Analítica da Universidade Federal do Ceará pelas análises de microscopia de varredura eletrônica (UFC/CT-INFRA/MCTI-SISNANO/Pró-equipamentos-CAPES). Este trabalho foi parcialmente financiado por meio de recursos da Coordenação de Aperfeiçoamento de Pessoal de Nível SuperiorCAPES (PROEX 23038.000509/2020-82).

\section{REFERÊNCIAS}

1. Mahahan, S. E.; Environmental Chemistry, 10 $0^{\text {th }}$ ed., CRC Press: New York, 2017.

2. Subramani, A.; Jacangelo, J. G.; Water Res. 2015, 75, 164.

3. Marinoski, A. K.; Rupp, R. F.; Ghisi, E.; J. Environ. Manage. 2018, 206, 28.

4. Santhosh, C.; Velmurugan, V.; Jacob, G.; Jeong, S. K.; Grace, A. N.; Bhatnagar, A.; Chem. Eng. J. (Lausanne) 2016, 306, 1116.

5. Shannon, M. A.; Bohn, P. W.; Elimelech, M.; Georgiadis, J. G.; Marinas, B. J.; Mayes, A. M.; Nature 2008, 452, 301.

6. Figaroa, P. A.; Miedema, H.; Euverink, G. J.; Picchioni, F.; Pure Appl. Chem. 2017, 89, 51

7. Rocha, L. S.; Nogueira, J.; Daniel-Da-Silva, A. L.; Marques, P.; Fateixa, S.; Pereira, E.; Trindade, T.; J. Environ. Chem. Eng. 2021, 9, 105405.

8. Qin, C.; Wang, R.; Ma, W.; Desalination 2010, 259, 156.

9. Zewail, T. M.; Yousef, N. S.; Alexandria Eng. J. 2015, 54, 83.

10. El-Nahas, S.; Osman, A. I.; Arafat, A. S.; Al-Muhtaseb, A. H.; Salman, H. M.; J. Water Process. Eng 2020, 33, 101104.

11. Cundy, C. S.; Cox, P. A.; Chem. Rev. 2003, 103, 663.

12. Luna, F. J.; Schuchardt, U.; Quim. Nova 2001, 24, 885.
13. Collins, F.; Rozhkovskaya, A.; Outram, J. G.; Millar, G. J.; Microporous Mesoporous Mater. 2020, 291, 109667.

14. Herrmann, C. C.; Klein, G.; React. Polym., Ion Exch., Sorbents 1987, 5, 281.

15. Koohsaryan, E.; Anbia, M.; Maghsoodlu, M.; J. Environ. Chem. Eng. 2020, 8, 104287.

16. Grecco, S. D. T. F.; Rangel, M. D. C.; Urquieta-Gonzalez, E. A.; Quim. Nova 2013, 36, 131.

17. Yang, X.; Liu, Y.; Yan, C.; Chen, G.; J. Cryst. Growth 2019, 528, 125286.

18. Anis, S. F.; Khalil, A.; Saepurahman; Singaravel, G.; Hashaikeh, R.; Microporous Mesoporous Mater. 2016, 236, 176.

19. Anis, S. F.; Singaravel, G.; Hashaikeh, R.; RSC Adv. 2018, 8, 16703.

20. Chen, L. H.; Sun, M. H.; Wang, Z.; Yang, W.; Xie, Z.; Su, B. L.; Chem. Rev. (Washington, DC, U. S.) 2020, 120, 11194.

21. Schwieger, W.; Machoke, A. G.; Weissenberger, T.; Inayat, A.; Selvam, T.; Klumpp, M.; Inayat, A.; Chem. Soc. Rev. 2016, 45, 3353.

22. Okada, K.; Kuboyama, K.; Takei, T.; Kameshima, Y.; Yasumori, A.; Yoshimura, M.; Microporous Mesoporous Mater. 2000, 37, 99.

23. Wardani, A. R. K.; Widiastuti, N.; Indones. J. Chem. 2016, 16, 1.

24. Wu, Z.; Xie, J.; Liu, H.; Chen, T.; Cheng, P.; Wang, C.; Kong, D.; Adsorpt. Sci. Technol. 2018, 36, 1700.

25. Tabi, R. N.; Agyemang, F. O.; Mensah-Darkwa, K.; Arthur, E. K.; Gikunoo, E.; Momade, F.; Mater. Chem. Phys. 2021, 261, 124229.

26. Faghihian, H.; Moayed, M.; Firooz, A.; Iravani, M.; J. Colloid Interface Sci. 2013, 393, 445.

27. Bessa, R. A.; Costa, L. S.; Oliveira, C. P.; Bohn, F.; Nascimento, R. F.; Sasaki, J. M.; Loiola, A. R.; Microporous Mesoporous Mater. 2017, 245 , 64.

28. Loiola, A. R.; Andrade, J. C. R. A.; Sasaki, J. M.; Da Silva, L. R. D.; J. Colloid Interface Sci. 2012, 367, 34.

29. El-Nahas, S.; Osman, A. I.; Arafat, A. S.; Al-Muhtaseb, A. A. H.; Salman, H. M.; J. Water Process. Eng 2020, 33, 101104.

30. Rahman, A. U.; Khan, F. U.; Rehman, W. U.; Saleem, S.; J. Chem. Technol. Metall. 2018, 53, 825 .

31. Song, J.; Liu, M.; Zhang, Y.; AIChE J. 2015, 61, 640.

32. Feitosa, F. A. C.; Hidrogeologia: conceitos e aplicações, $3^{\mathrm{a}}$ ed., CPRM: Rio de Janeiro, 2008.

33. Ghadamnan, E.; Nabavi, S. R.; Abbasi, M.; J. Water Environ. Nanotechnol. 2019, 4, 119.

34. Nightingale Jr., E. R.; J. Phys. Chem. 1959, 63, 1381.

35. Nasief, F. M.; Shaban, M.; Alamry, K. A.; Khadra, M. R. A.; Khan, A. Aa. P.; Asiri, A. M.; El-Salam, H. M. A.; J. Environ. Chem. Eng. 2021, 9, 105834. 